\title{
A novel domain overlapping strategy for the multiscale coupling of CFD with 1D system codes with applications to transient flows.
}

\author{
T. P. Grunloh and A. Manera \\ Department of Nuclear Engineering and Radiological Sciences, University of Michigan-Ann Arbor, MI \\ 2355 Bonisteel Blvd., Ann Arbor, MI 48109, USA \\ grunloh@umich.edu,manera@umich.edu
}

\begin{abstract}
A novel multiscale coupling methodology based on a domain overlapping approach has been developed to couple a computational fluid dynamics code with a best-estimate thermal hydraulic code. The methodology has been implemented in the coupling infrastructure code Janus, developed at the University of Michigan, providing methods for the online data transfer between the commercial computational fluid dynamics code STAR-CCM+ and the US NRC best-estimate thermal hydraulic system code TRACE. Coupling between these two software packages is motivated by the desire to extend the range of applicability of TRACE to scenarios in which local momentum and energy transfer are important, such as three-dimensional mixing. These types of flows are relevant, for example, in the simulation of passive safety systems including large containment pools, or for flow mixing in the reactor pressure vessel downcomer of current light water reactors and integral small modular reactors. The intrafluid shear forces neglected by TRACE equations of motion are readily calculated from computational fluid dynamics solutions. Consequently, the coupling methods used in this study are built around correcting TRACE solutions with data from a corresponding STAR-CCM+ solution.
\end{abstract}

Two coupling strategies are discussed in the paper: one based on a novel domain overlapping approach specifically designed for transient operation, and a second based on the well-known domain decomposition approach. In the present paper, we discuss the application of the two coupling methods to the simulation of open and closed loops in both steady state and transient operation. The objective of this study is to examine the performance of each coupling method in terms of convergence, consistency, and numerical stability.

As expected, the results produced by the two methods were found to be identical, once numerical convergence is achieved, and consistent with the standalone STAR-CCM+ solution in both steady state and transient cases. However, the domain overlapping method was found to achieve convergence at larger integration time steps than the domain decomposition approach and exhibited superior convergence and numerical stability characteristics in both steady state and transient scenarios.

KEYWORDS coupling, separate, decomposition, overlapping, TRACE, CFD

\section{INTRODUCTION}

\subsection{Motivation}

Although currently employed computational techniques have shown a remarkable ability to simulate the complex behavior of a Nuclear Power Plant (NPP) under a wide range of conditions, current trends of 
economic optimization of nuclear power plants (new designs, power uprates of operating reactors, etc.) motivate calculations of increasing fidelity for better estimates of safety margins. The proficiency of system thermal hydraulic (STH) codes (i.e. TRACE [1], RELAP5 [2], CATHARE [3], and ATHLET [4]) in the simulation of large networks of interconnected NPP components has been well established and documented in an ample body of literature. When three-dimensional effects play an important role in the NPP system, the constitutive relations and simplifying assumptions upon which STH codes are based become invalid. Specifically, best-estimate system codes are unable to correctly capture circulating flow patterns in large open regions, such as inlet plena, which, for example, are important in boron dilution, main steam line break, pressurized thermal shock transients, and transients involving many safety passive systems under accident conditions.

In recent years, however, Computational Fluid Dynamics (CFD) methods have been increasingly applied to the simulation of single phase flow in complex geometries relevant to nuclear engineering. To name just a few, Höhne et al. [5] simulated the mixing of de-borated slugs of water through the ROCOM facility, Lee et al. [6] studied the effect of PWR internals on core inlet flow, and Jeong et al. [7] simulated the flow field in downcomer and lower plenum of a PWR, explicitly accounting for reactor internals. The enduring trend of increasing availability of computational resources continues to add larger, higher resolution models to the class of tractable problems. Furthermore, researchers such as Merzari and Ninokata [8] and Prill and Class [9] have explored the use of Proper Orthogonal Decomposition (POD) to develop Reduced Order Models (ROM) for NPPs, potentially reducing the runtime computational burden significantly. Therefore, the coupling of CFD (or CFD-like ROMs) codes with best-estimate thermalhydraulic system codes is a worthwhile endeavor, particularly in view of current industry inclinations towards power uprates, with the consequent reduction of safety margins, mandating a compensating increase in the fidelity of predictive models to satisfy safety regulations.

Primarily, two spatial decomposition methods are described in the literature, referred to as the domain decomposition and domain overlapping approaches. In the former, the system to be modeled is divided into two discrete domains: one simulated using the system code and the other computed within the CFD code, connected through data exchange at the domain interfaces. In the domain overlapping approach, the entire system is computed within the system code, with selected regions also simulated within the CFD code. The first coupling efforts for nuclear applications reported in the literature were from Aumiller et al. [10] and Gibeling and Mahaffy [11], both works based on domain decomposition approaches. In recent years, several additional efforts have been focused on similar approaches. Anderson et al. [12] analyzed the Very High Temperature Reactor (VHTR). Bertolotto et al. [13] performed single phase mixing studies. Papukchiev et al. [14] coupled ATHLET and ANSYS CFX and applied it to a Pressurized Thermal Shock Transient [15]. Watanabe et al. [16] studied steam generator instability during station blackout conditions. Li et al. [17] coupled FLUENT and RELAP5 to study the Edwards-O'Brien blowdown problem as well as multi-loop flow through a 3D region. In some cases [10] [13] [14] [15], semi-implicit numerical coupling schemes are also described, aimed at relaxing the requirements on the integration time steps posed by explicit time-stepping methods, at the expense of increased computational costs. Additionally, implicit or semi-implicit methods generally require more data storage and stronger manipulation of each code's execution due to time step backups.

Researchers have employed domain overlapping methods targeted at various phenomena. Fanning and Thomas [18] used a CFD solution to correct the energy equation and the gravitational pressure term computed by the system code. Jeltsov et al. [19] used a CFD solution to correct the system code energy equation through the implementation of a "virtual heater". Baviere et al. [20] [21] used a CFD solution to correct both momentum and enthalpy equations using iterative controls. In this case, a source/sink term in the momentum equation of the system code CATHARE is iteratively adjusted within a given time step until the overall pressure drop across the overlapped component is identical to the CFD solution. 
A more direct coupling approach is to modify the closure coefficients used by the system code, taking advantage of pre-existing functionality of the code. Cadinu and Kudinov [22] employed such a method, but did not explicitly account for inertial effects on the pressure gradient, limiting transient applicability. The question therefore arises of how to couple a system code to a CFD code that performs well when inertial pressure effects are not negligible. We address this by deconstructing the pressure gradient to account for inertial effects and calculate a transient-compatible closure relation for the system code based on real-time CFD data.

Comparisons among coupled CFD/STH simulations based on overlapping and domain decomposition approaches have been reported in the literature. Papukchiev et al. [23] compared coupled approaches to simulations of the TALL-3D experiment and Bandini et al. [24] assessed several coupling implementations based on their application to Generation IV reactor concepts. However, because different coupled codes were used (RELAP5/STAR-CCM+, ATHLET/ANSYS CFX, CATHARE/TRIO-U), these comparisons cannot be used to draw generalized conclusions on the performance of a given coupling scheme versus another. Consequently, the work presented in this paper further undertakes a systematic comparison of domain overlapping and domain decomposition methods to assess their relative performance. The results show that the novel domain overlapping method proposed here is able to successfully correct the solution of the system code TRACE based on the solution of the CFD code STAR-CCM+. In addition, it is found that for the cases tested, the proposed domain overlapping method exhibits superior stability and temporal convergence characteristics as compared to a domain decomposition implementation.

\section{Theory}

\subsection{Domain Decomposition Approach}

In a domain decomposition approach, the modeled system is divided into two (or more) discrete domains, each simulated either within the system code or within the CFD code. Domain decomposition coupling is then realized through the boundary data transfer between the region(s) modeled with the system code and the region(s) modeled with the CFD code. A schematic of the specific domain decomposition coupling strategy employed in this study is shown in Figure 1. A domain decomposition interface can connect a TRACE BREAK (pressure boundary condition) component to a STAR-CCM+ inlet boundary (Figure 1 left), or a TRACE FILL (mass flow or velocity boundary condition) component to a STAR-CCM+ pressure outlet boundary (Figure 1 right). In the case of the former, the pressure calculated at the inlet of the CFD region is passed to the cell center of the BREAK component and the flow rate in the BREAK is passed to the CFD inlet. In the case of the latter, the pressure at the center of the cell nearest the interface is passed to the CFD outlet boundary while the flow rate through the CFD outlet is passed to the FILL component.
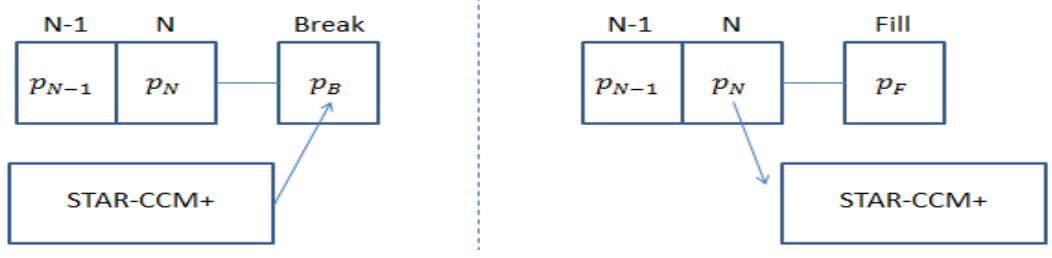

Figure 1. Schematic diagram of data exchange between boundaries of TRACE and STAR-CCM+ for domain decomposition coupling

STH codes like TRACE often employ a staggered grid arrangement with thermodynamic quantities like pressure and temperature defined in the center of control volumes $(\mathrm{CV})$ and have the physical meaning of averages over the CV. Flux quantities such as velocity are defined at the boundaries of the CV. In such cases, interpolation is necessary to calculate the velocity at the center of a $\mathrm{CV}$ or the pressure at the $\mathrm{CV}$ 
boundary. Using a surface pressure computed by the CFD code, as is performed here, to modify the pressure in the system code coupled node can therefore introduce some error. While this error is often negligible in practice, some STH codes extrapolate thermodynamic variables to the CV edge. Additionally node lengths and form loss coefficients can be adjusted to meet requirements. In the case presented in $\$ 3.3$., we employ both interfaces shown in Figure 1 to remove the region from a closed loop. With this configuration, we benefit from error cancellation since one interface effectively adds length to the TRACE component (Figure 1 left) while the other removes length (Figure 1 right).

The primary benefit of domain decomposition coupling lies in the simplicity of implementation and theoretical framework. A pressure drop imposed on TRACE with domain decomposition coupling in both transient and steady state is given by Eq. 1, which suggests perfect pressure consistency between the two codes in all cases.

$$
\Delta p_{T R C}=\Delta p_{C F D}
$$

\subsection{Domain Overlapping Approach}

In the domain overlapping coupling paradigm, the entire flow domain is simulated with the system code, while a selected portion of the domain is also simulated with the CFD code. The codes are then volumetrically coupled in the overlapping region. The system code (TRACE) solution is corrected internally based on data from the CFD code (STAR-CCM+). The correction is motivated by the assumption that in the selected overlapped regions, the CFD solution will be more accurate than the system code solution. A properly validated CFD model is therefore a prerequisite to the appropriate application of this coupling algorithm. This coupling methodology allows the system code to solve the mass and momentum balance equations for the entire system and prevent mass conservation inconsistencies that can lead to numerical instabilities for closed loop simulations [14].

A simplified version of the one-dimensional equation of motion implemented in TRACE is given in Eq. 2. Here the superscript $n$ refers to the old time step solution, $(n+1)$ to the new time step, $V_{j+1 / 2}^{n+1}$ refers to the velocity at the new time step defined at the edge between cells $j$ and $j+1$, while $\langle\rho\rangle_{j+\frac{1}{2}}^{n}$ refers to the average liquid density between nodes $j$ and $j+1, p$ is node averaged pressure, $\Delta x$ is the distance between centers of neighboring cells, and $K_{j+1 / 2}^{n}$ is a coefficient used to model the effects of both form and frictional loss. For the purposes of this study, we focus on the frictional aspect of the coefficient.

$$
\frac{V_{j+1 / 2}^{n+1}-V_{j+1 / 2}^{n}}{\Delta t}+\left.V_{j+1 / 2}^{n} \frac{\partial V^{n+1}}{\partial x}\right|_{j+1 / 2}=-\frac{1}{\langle\rho\rangle_{j+\frac{1}{2}}^{n}} \frac{p_{j+1}^{n+1}-p_{j}^{n+1}}{\Delta x}-K_{j+1 / 2}^{n}\left[2 V_{j+1 / 2}^{n+1}-V_{j+1 / 2}^{n}\right]\left|V_{j+1 / 2}^{n}\right|
$$

Comparison to the Navier-Stokes momentum balance equations shows that the divergence of the stress tensor $\mathbf{T}$ in a system code like TRACE is modeled as proportional to the square of a velocity. The constant of proportionality is calculated using the Churchill correlation for the Fanning friction factor [1].

$$
-\frac{\nabla \cdot \mathbf{T}}{\rho} \approx K V^{2}=2 f_{F} \frac{\Delta x}{D_{h}} V^{2}
$$

Based on this analysis, a CFD based friction factor can be calculated from the definition of the Fanning friction factor [25], as shown in Eq. 4, similar to the treatment used by other researchers [22]. 


$$
f_{C F D}=\frac{1}{2} \frac{D_{h}}{\rho_{T R C}\left(V_{j+1 / 2}^{n}\right)^{2}} \frac{\Delta p_{C F D}}{L_{L P}}
$$

The CFD friction factor defined in Eq. 4 is computed from the global pressure drop across the CFD component, $\Delta P_{C F D}$. Because this pressure drop includes the convective pressure drop calculated in CFD, the associated term (form loss contribution to $K$ in Eq. 2) is eliminated from the TRACE momentum equation coefficients associated only with the coupled (overlapped domain) component. Division by a lumped parameter (LP) length scale $L_{L P}$ yields an average pressure gradient over the overlapped component. The length scale $L_{L P}$ is the length of the component as defined in TRACE and is automatically retrieved from the TRACE input data by the coupling interface. The average pressure gradient is then normalized by the hydraulic diameter $\left(D_{h}\right)$ and the local TRACE values for density $\left(\rho_{T R C}\right)$ and old time step velocity $\left(V_{j+1 / 2}^{n}\right)$.

Combining Equations 2, 3, and 4, for steady state conditions, the pressure drop across the $j+1 / 2$ face is calculated as shown in Eq. 5. Thus, the pressure drop across an edge (i.e. between adjacent CVs) is simply the total CFD pressure drop scaled down to the correct length for edge $j+1 / 2$.

$$
\Delta p_{j+1 / 2}=-\operatorname{sign}\left(V_{j+\frac{1}{2}}\right) \Delta p_{C F D}\left(\frac{\Delta x}{L_{L P}}\right)
$$

For transient simulations, the inertial term of the momentum equation cannot be neglected. The transient pressure drop between node $j$ and $j+1$ for TRACE is shown in Eq. 6, which includes, in addition to the contribution of the CFD pressure drop, an inertial contribution. The CFD pressure drop also includes an inertial contribution, leading to an overestimation of these effects and inconsistency between CFD and STH.

$$
\Delta p_{j+1 / 2}=-\rho \Delta x \frac{V_{j+\frac{1}{2}}^{n+1}-V_{j+\frac{1}{2}}^{n}}{\Delta t}-\Delta x \frac{1}{\left(V_{j+\frac{1}{2}}^{n}\right)^{2}} \frac{\Delta p_{C F D}^{n}}{L_{L P}}\left(2 V_{j+\frac{1}{2}}^{n+1}-V_{j+\frac{1}{2}}^{n}\right)\left|V_{j+\frac{1}{2}}^{n}\right|
$$

Consistency between CFD and system code can be achieved by computing a non-inertial pressure gradient based friction factor through appropriate modification of $\Delta p_{C F D}^{n}$. Starting from the Navier-Stokes equations, the pressure gradient can be partitioned into three distinct terms as shown in in Eq. 7. The first term on the right, the inertial pressure contribution, results from unsteady velocities. The second term on the right, the convective pressure gradient, results from acceleration due to velocity gradients. Finally, the frictional pressure gradient resulting from viscous dissipation is the third term on the right. In cases of turbulent flow, consider $\mathbf{v}$ to be the Reynolds averaged velocity and for $\mathbf{T}$ to include Reynolds stresses.

$$
\nabla p=-\rho \frac{\partial \mathbf{v}}{\partial t}-\rho \mathbf{v} \cdot \nabla \mathbf{v}+\nabla \cdot \mathbf{T}
$$

In an explicit operator splitting coupling scheme, it is not straightforward to directly correct for the inertial pressure term in the STH code, since this term results from velocity updates at each time step. However, since we are limiting the coupling to regions where the flow is incompressible, it is justified to assume that the difference between the inertial pressure drops as computed separately by CFD and the system code for the overlapped domain is negligible as long as fluid velocities are consistent. Therefore, only the non-inertial pressure drop terms (acceleration and friction) need to be corrected. In order to cast the CFD solution into a form that can readily be used to correct the TRACE solution in the overlapped domain, a volume averaged non-inertial (NI) pressure gradient is defined and $\Delta p_{C F D}^{n}$ in Eq. 6 is replaced 
with an NI version, shown in Eq. 8 to be the total pressure gradient with the inertial contribution subtracted.

At this point, it is convenient to focus on assumptions specific to 1D pipe type components. Such test cases provide convenient grounds for establishing proof-of-principle calculations. A more general interface applicable to 3D geometries, such as open regions with recirculation, based on the same foregoing principles has been developed and will be presented in a future paper.

In order to condense the pressure gradient vector field into a scalar field, the integrand of Eq. 8 is dotted with a flow path vector $\widehat{\boldsymbol{n}}_{f p}$. This vector field is related to the direction of the mean flow at each cross section and can be user input or derived from geometric input data available to the STH code for 1D components.

$$
\left\langle\nabla p \cdot \widehat{\boldsymbol{n}}_{f p}\right\rangle_{N I}=\left\langle\nabla p \cdot \widehat{\boldsymbol{n}}_{f p}\right\rangle_{t o t}-\left\langle\nabla p \cdot \widehat{\boldsymbol{n}}_{f p}\right\rangle_{I}=\left\langle\nabla p \cdot \widehat{\boldsymbol{n}}_{f p}\right\rangle_{t o t}+\left\langle\rho \frac{\partial \mathbf{v}}{\partial t} \cdot \widehat{\boldsymbol{n}}_{f p}\right\rangle
$$

The volume averaged $\Delta p$ can then be related to the total change in pressure $p$ between two surfaces:

$$
\left\langle\nabla p \cdot \widehat{\boldsymbol{n}}_{f p}\right\rangle_{t o t}=-\frac{\Delta p}{L}
$$

where $\Delta p$ in this case refers to the change in surface averaged pressures between the surfaces that bound the averaging volume and have normal vectors parallel to $\widehat{\boldsymbol{n}}_{f p}$ and $L$ is the length of this volume (with volume $\mathrm{Vol}$ ) as modeled in TRACE. The sign convention is defined such that $\Delta p=p_{\text {inlet }}-p_{\text {outlet }}$ is the difference between the inlet and outlet of a 1D geometry and $\Delta p>0$ for steady state flow through a straight pipe. By combining Eqs. 4, 8, and 9, a friction factor based on CFD data can be defined for the correction of the TRACE momentum equation, given by Eq. 10 in which $\mathbf{v}$ refers to the velocity vector field of the CFD solution.

$$
f_{C F D}=\frac{1}{2} \frac{D_{h}}{\rho_{T R C}\left(V_{j+1 / 2}^{n}\right)^{2}}\left[\frac{\Delta p_{C F D}^{n+1}}{L_{L P}}-\frac{1}{\Delta t}\left(\frac{1}{V o l} \iiint \rho \mathbf{v}^{n+1} \cdot \widehat{\boldsymbol{n}}_{f p} d V-\frac{1}{V o l} \iiint \rho \mathbf{v}^{n} \cdot \widehat{\boldsymbol{n}}_{f p} d V\right)\right]
$$

This friction factor requires three integrals to be evaluated at each time step for each coupled component: 1) inlet surface average pressure, 2) outlet surface average pressure, and 3) volume averaged velocity. The old time $\left(t^{n}\right)$ velocity integral must also be saved. As a point of clarification, the reader is reminded that Eq. 10 is only applicable to pipe geometries in which $\widehat{\boldsymbol{n}}_{f p}$ and $L_{L P}$ are easily defined. The extension to cases in which these definitions are not straightforward, such as in 3D open mixing regions, will be addressed in a future paper.

Currently, only explicit coupling has been implemented in the Janus infrastructure for transient simulations. Individually, STAR-CCM+ uses the "Implicit Unsteady" temporal solver, while TRACE uses the Stability Enhancing Two Step (SETS) solver by default [1] [26]. The coupling scheme used for the present analysis entails TRACE first calculating a timestep from time $t^{n}$ to $t^{n+1}$, then passing the new time data to STAR-CCM+ which then takes the same time step. The inverse of this was also tested and found to have similar accuracy.

\section{RESULTS}

In the previous section, we predicted that domain overlapping methods would allow for larger timesteps. In this section, we present results from three test cases representative of a wide range of potential 1D flow scenarios. In the first test case, we examine a simple 1D pipe case followed by a case in which the flow path vector $\widehat{\boldsymbol{n}}_{f p}$ is nontrivial. Finally, we examine a closed loop test case that includes additional feedback effects. 


\subsection{Open Loop Test Case}

Towards the comparison of domain decomposition and domain overlapping coupling, the methods are first applied to a simple case that lends itself to analytical treatment. Consider the geometry in Figure 2 in which a pipe of length $L$ is broken into two sections of lengths $L_{1}$ and $L_{2}$. For $t<0 \mathrm{~s}$, the pipe is stagnant with $p_{\text {in }}=p_{\text {out }}=1.0 \mathrm{MPa}$. Immediately after $t=0 \mathrm{~s}$, the outlet pressure drops and the total pressure drop across the pipe is held constant at $\Delta p=10 \mathrm{kPa}$ for $t>0 \mathrm{~s}$.

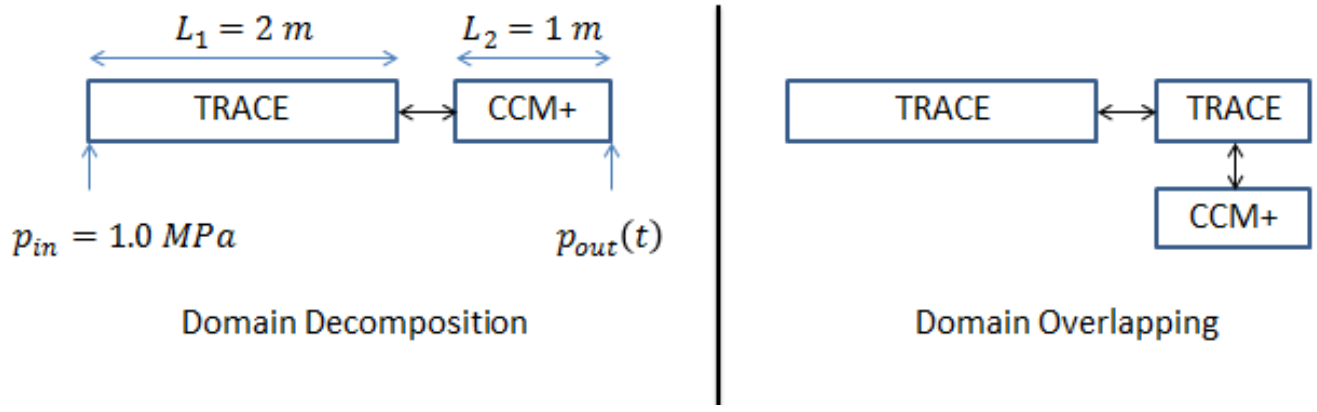

Figure 2. Schematic diagrams of the abrupt pressure drop test case coupling configurations.

The abrupt pressure drop case was previously explored by Bertolotto [27], who utilized a 3 meter pipe with a radius of 0.025 meters. We reproduced this test case with the first 2 meters of the pipe simulated by TRACE while the remaining meter is coupled to STAR-CCM+ through either domain decompositions (Figure 2 left) or domain overlapping (Figure 2 right) methods. The flow in the pipe is initially stagnant and at a constant pressure of $p_{\text {init }}=1.0 \mathrm{MPa}$. At time $t=0.0 \mathrm{~s}$, the pressure at the outlet is abruptly lowered to $p_{\text {out }}(t \geq 0.0 \mathrm{~s})=0.99 \mathrm{MPa}$.

The time dependent mass flow rates resulting from the abrupt pressure drop are shown in Figure 3 for both coupling methods. Additionally, the data calculated by Bertolotto with semi-implicit domain decomposition coupling using ANSYS CFX/TRACE are plotted as a reference. Convergence is observed with larger time steps when domain overlapping is used. In addition, significant mass flow overshoots are observed for the domain decomposition approach (Figure 3 bottom). These overshoots are present for all timesteps tested as shown in Figure 4, with the underlying cause discussed below. It is observed that the domain overlapping case converges with $\Delta t=0.1 \mathrm{~s}$ while the domain decomposition method requires an integration time step close to $\Delta t=0.0025 \mathrm{~s}$. Therefore, a significant savings in terms of integration time step, and hence computational resources requirements, is realized. 

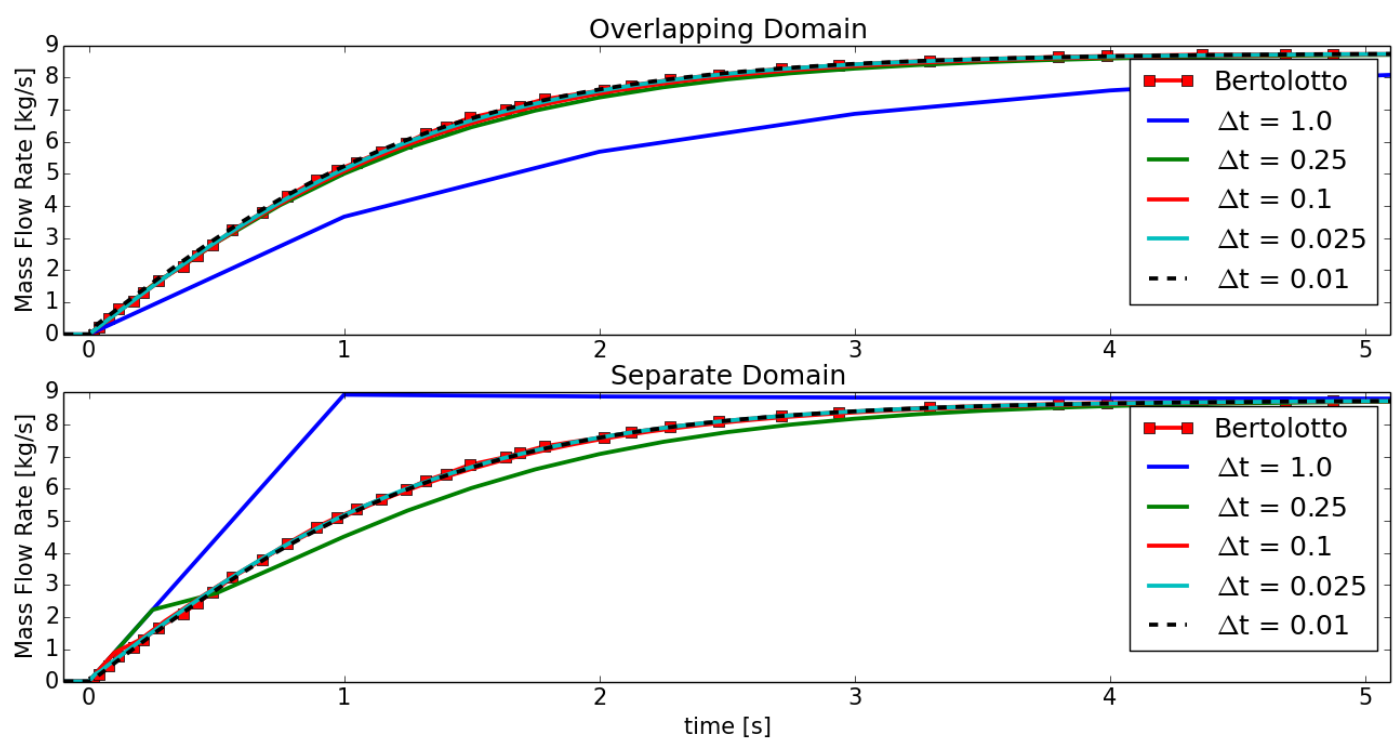

Figure 3. Mass flow rates through the open loop as a function of time for both coupling methods.

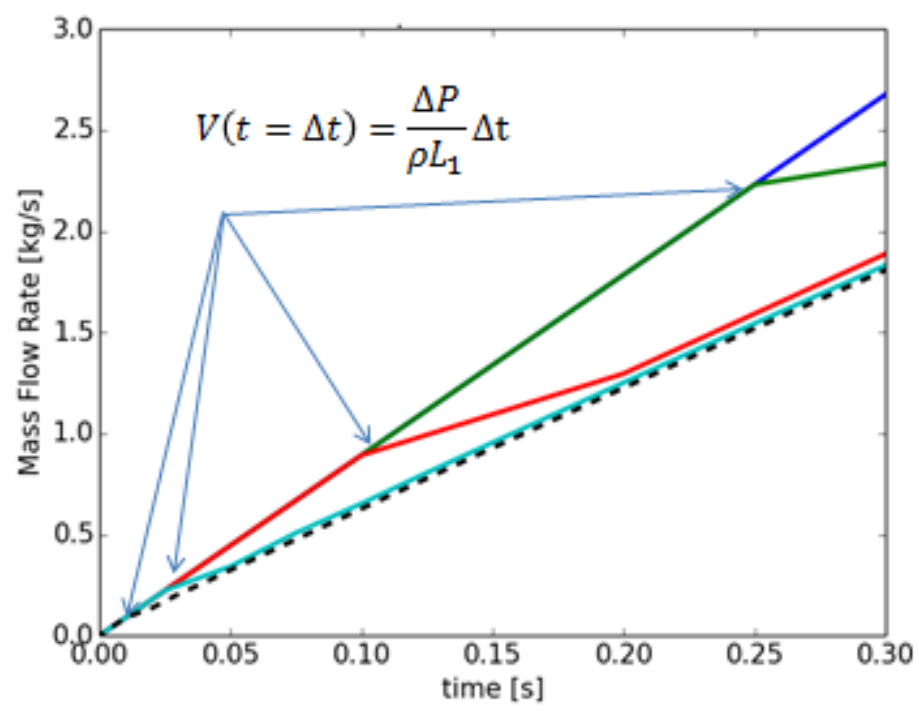

Figure 4. Zoomed picture of domain decomposition mass flow rates from Figure 3 to show overshoots.

In order to understand the cause of the mass flow overshoots in domain decomposition, it is useful to consider an accompanying simplified analytical model. Because the pipe cross section does not change along the pipe axis, the only contributions to the pressure drop are given by the inertial $\left(\Delta p_{I}\right)$ and frictional $\left(\Delta p_{f}\right)$ terms of the momentum equation. Utilizing the Fanning friction factor to model the frictional terms yields Eq. 11 as a model governing equation of the system, where $L=L_{1}+L_{2}$ is the total length of the system as shown in Figure 2.

$$
\Delta p=10 k P a=\Delta p_{f}+\Delta p_{I}=2 f_{F} \frac{L}{D} \rho V^{2}+L \rho \frac{\partial V}{\partial t}
$$

At the beginning of the transient, the pressure drop is dominated by the inertial term, so that the initial velocity gradient can be estimated using Eq. 12 . 


$$
\left.\frac{\partial V}{\partial t}\right|_{t=0}=\frac{\Delta p}{\rho L}
$$

It is useful to apply Eq. 12 to the first iterative cycle of an explicit domain decomposition coupled calculation. Before any iteration occurs, the mass flow rate of STAR-CCM+ is set to zero and the pressure everywhere is uniform. The pressure of the outlet (STAR-CCM+) is then lowered abruptly, but the mass flow rate is still everywhere zero due to the CFD inlet relying on TRACE for mass flow. The STAR-CCM+ outlet pressure is thus translated to the outlet of the TRACE section. TRACE then calculates the new velocity based on the entire pressure drop but only across the TRACE portion of the pipe as estimated in Eq. 13 .

$$
\left.\frac{\partial V}{\partial t}\right|_{t=0}=\frac{\Delta p}{\rho L_{1}}>\frac{\Delta p}{\rho L}
$$

Thus, it is expected that an explicit domain decomposition coupling will overestimate the velocity at the first timestep by a factor of $L / L_{1}$, which is 1.5 in the case of the geometry in Figure 2. In the domain overlapping coupling, the governing equation of the 1D system is Eq. 14, in which the Fanning friction factor has been replaced with the factor calculated from CFD data.

$$
10 k P a=2 f_{C F D} \frac{L}{D} \rho V^{2}+L \rho \frac{\partial V}{\partial t}
$$

Because the entire length of the component is available to TRACE in this case, the effect of Eq. 13 does not occur. While generally $f_{F} \neq f_{C F D}$, the two factors are expected to be of the same magnitude, at least to such an extent as to not significantly alter the mathematical structure of the governing equation. Thus, it is concluded that, for reasonable $f_{C F D}$, the coupled solution will evolve in a geometrically consistent manner $\left(\left.\frac{\partial V}{\partial t}\right|_{t=0}=\frac{\Delta p}{\rho L}\right)$. The analysis of this simple system suggests that, in the case of abrupt pressure drops, the domain overlapping method will outperform the domain decomposition method as long as $f_{C F D} \sim f_{F}$. While the instantaneous pressure drop is a special case, continuously changing systems become abrupt under temporal discretization. Thus, the domain overlapping method appears to better suited for larger timesteps, potentially allowing for improved computational efficiency.

\section{2. $\quad$ Bent Pipe Test Case}

The geometry of the previous test case was modified to include two bends (Figure 5). In this case the CFD solution does not present a radial symmetry as for the case of a straight pipe. In addition, the presence of a bend will give rise to localized frictional pressure drops, which in a 1D system code like TRACE are typically modeled with a user-defined form loss coefficient. The geometry of the test consists of a pipe of radius $0.03 \mathrm{~m}$ with two right angle turns with centerline curvature $0.07 \mathrm{~m}$. For the TRACE standalone simulations, a form factor $K=0.16$ was input at each bend. 


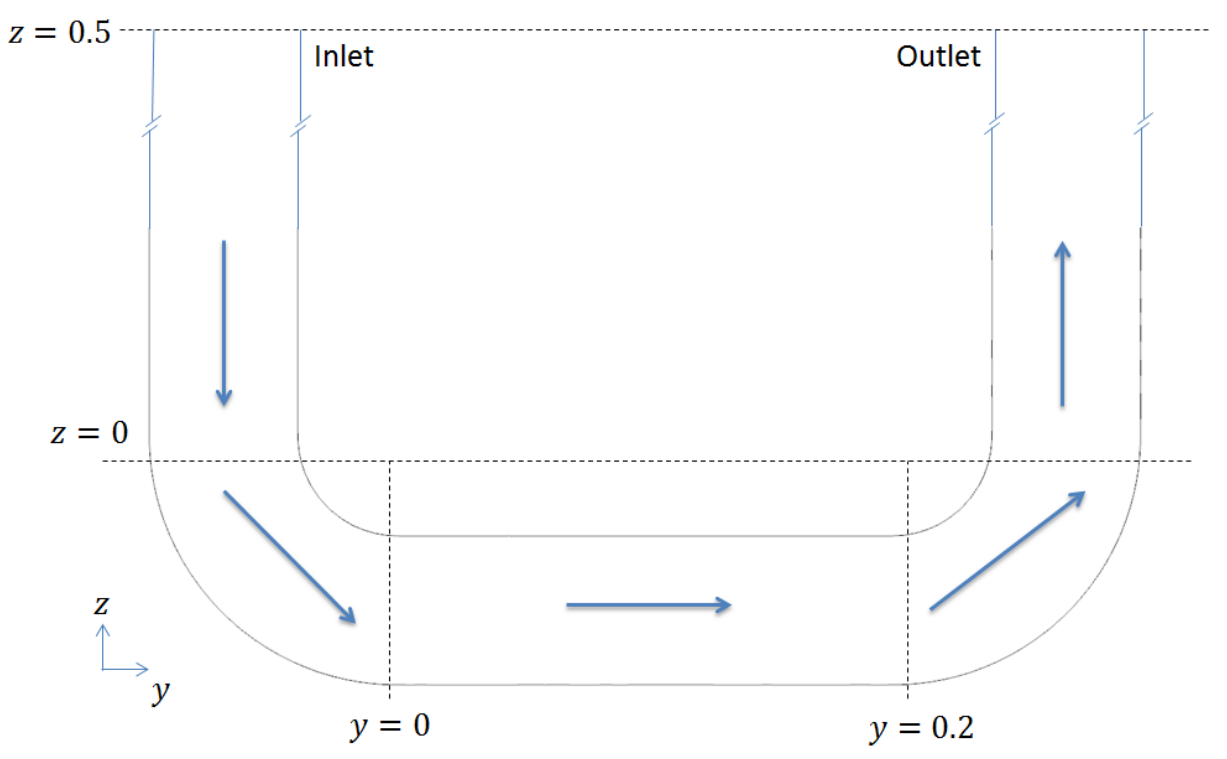

Figure 5. Schematic illustration of flow path vector field used for the bent pipe test case.

\subsubsection{Steady State}

Three simulations were run for this case: a TRACE standalone simulation, a STAR-CCM+ standalone simulation and a coupled TRACE/CCM+ simulation. The results obtained for the pressure drop across the component as function of the mass flow rate $\dot{m}_{\text {in }}$ in steady state conditions are reported in Figure 6 . The comparison shows that the coupled TRACE/CCM+ results are consistent with STAR-CCM+ standalone simulations, justifying Eq. 10 under steady state conditions.

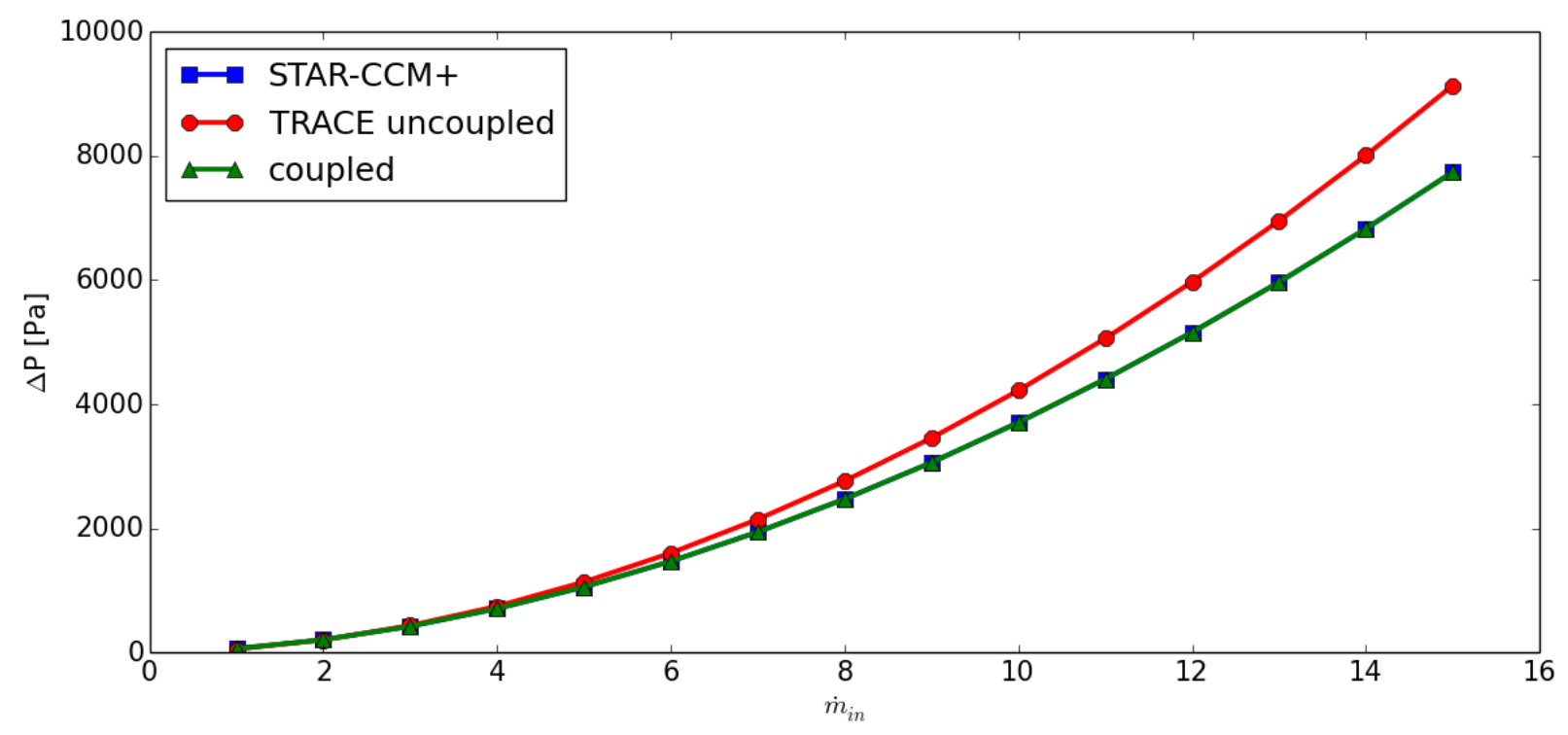

Figure 6. Steady state results for bent pipe coupled case.

\subsubsection{Transient}

The friction factor definition of Eq. 10 reduces to a simple form for steady state cases, requiring transient operation to engage the inertial correction. In pursuit of this, the time dependent mass flow rate of Eq. 15 was applied to the inlet of the bent pipe, with the resulting time dependent pressure drop recorded as output. 


$$
\dot{m}_{\text {in }}(t)=\left\{\begin{array}{cc}
10.0 \frac{\mathrm{kg}}{\mathrm{s}}, & t \leq 10.0 \mathrm{~s} \\
t \times 1.0 \frac{\mathrm{kg}}{\mathrm{s}^{2}}, & 10.0 \mathrm{~s}<t<15.0 \mathrm{~s} \\
15.0 \frac{\mathrm{kg}}{\mathrm{s}}, & t \geq 15.0 \mathrm{~s}
\end{array}\right.
$$

Data collected from a selected transient simulation $(\Delta t=0.1 \mathrm{~s})$ are displayed in Figure 7 (left). The coupling is activated at $t=9.0 \mathrm{~s}$. After a short null transient that allows equilibration between CCM+ and TRACE to, the transient mass flow rate begins. The on-the-fly correction of the TRACE solutions using CFD data is shown to be successful in transient conditions. Similarly to the steady case, the standalone TRACE simulation differs from the CFD pressure drop while the coupled solution closely follows the CFD solution.

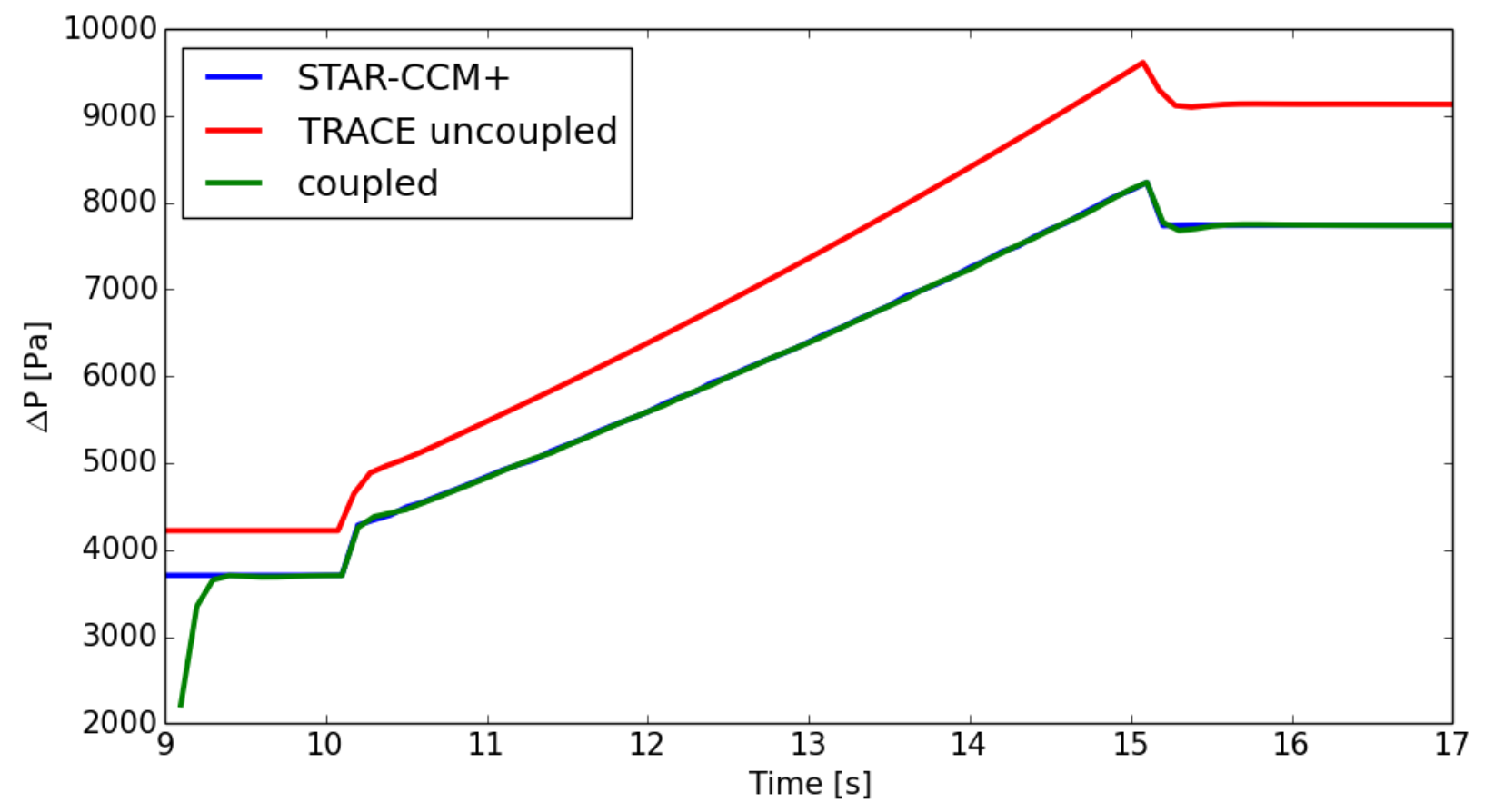

Figure 7. Transient bent pipe coupling test case. Calculated with $\Delta t=0.1 \mathrm{~s}$. 


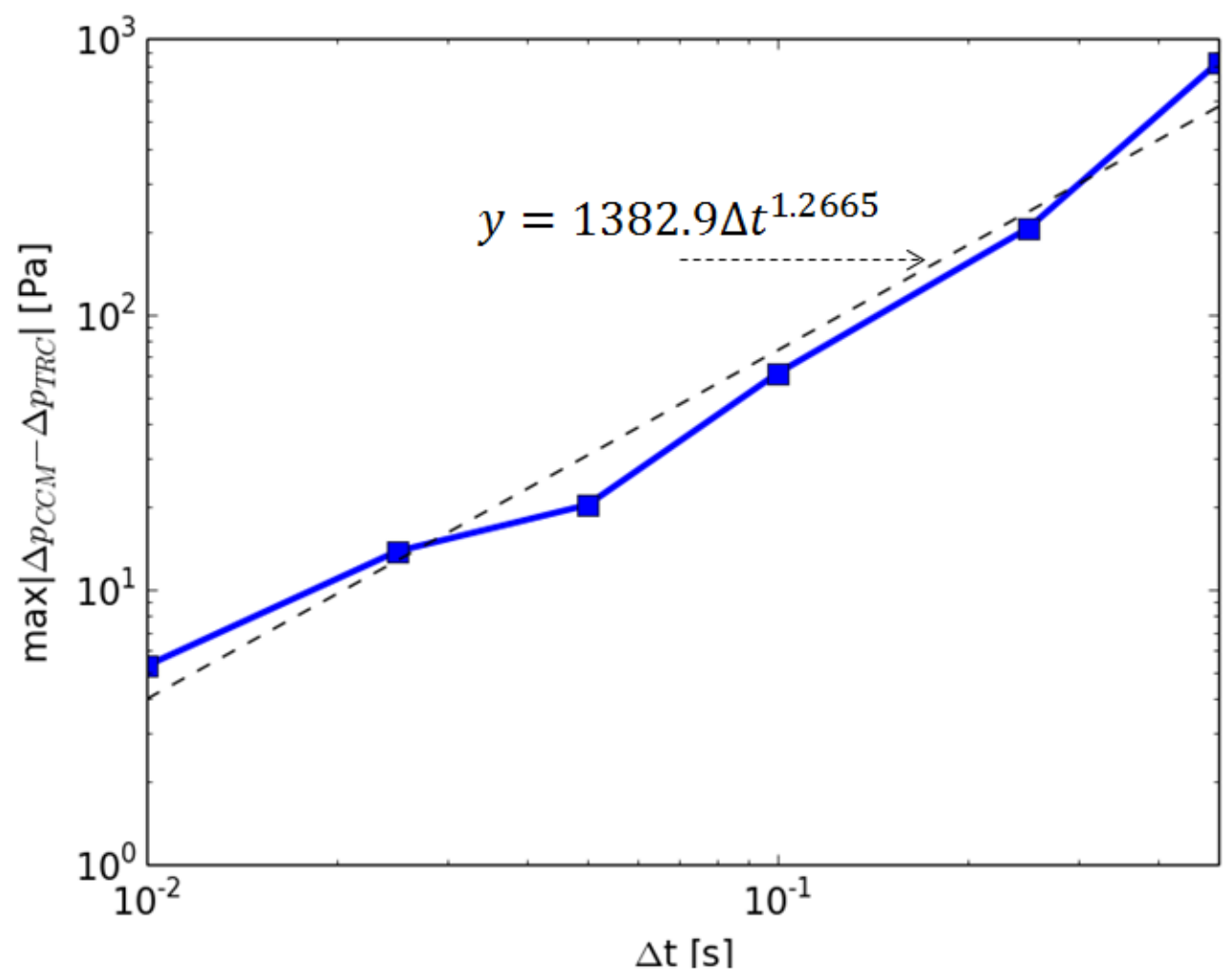

Figure 8. $L^{\infty}$ error in agreement between TRACE and STAR-CCM+ for domain overlapping coupling of the bent pipe test case with power law data fit to show approximately linear diminishing of error.

In Figure 7, some visible differences between the "STAR-CCM+" and the "coupled" curves are apparent. Because the codes calculate the inertial pressure drop independently, some error is introduced. We measure this error by calculating the maximum difference ( $L^{\infty}$ norm error) between the two curves after the initial null transient. This number is included in Figure 8 for several timesteps, where the error is observed to diminish approximately linearly with decreasing timestep.

\section{3. $\quad$ Pump Driven Closed Loop Test Case}

The test case is made progressively more challenging by closing the loop to include feedback effects. The loop considered in this section, as shown in Figure 9 (top), consists of three pipe sections, a pump, and a pressure set-point to prevent runaway pressures from building up within the loop. The pump is driven by a custom pump head curve designed to produce the desired mass flow rates. 


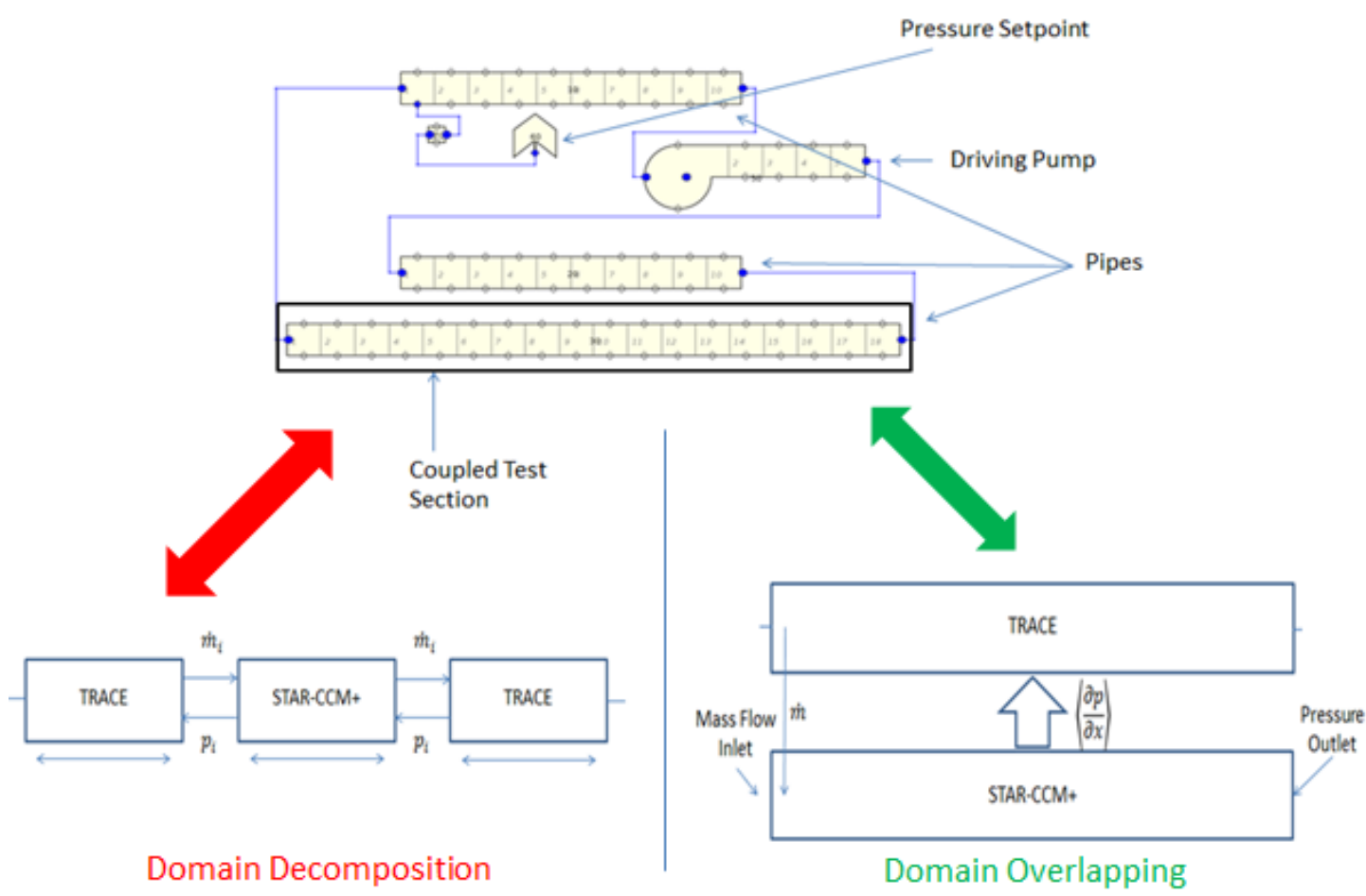

Figure 9. TRACE model of closed, pump driven loop with employed coupling schemes indicated. Both coupling schemes are used in separate calculations to replace the solution in the coupled test section with CFD-based results.

The coupled test section modeled with CFD is indicated in Figure 9 and consists of a $1.8 \mathrm{~m}$ long circular pipe with a diameter of $0.1 \mathrm{~m}$. For the domain overlapping approach, this section is also modeled within TRACE using 18 cells of equal length. The accompanying STAR-CCM+ component is a circular tube of the same dimensions as the TRACE pipe and is composed of approximately 23000 cells. True consistency between CFD and system code simulations would require fully developed velocity and turbulence profiles at the CFD inlet because TRACE assumes the fully developed flow state. However, for the current test case, flat inlet velocity profiles with default turbulence parameters are imposed at the CFD inlet. With this assumption, a given mass flow rate will result in a lower TRACE (standalone) pressure drop compared to CFD. The effects are observed to be quite small and thus it is deemed acceptable to test the coupling implementation.

\subsubsection{Interfacing}

The domain overlapping technique is applied through one volumetric interface connecting the test section in the complete TRACE model to the CFD model of the component as shown in Figure 9 (bottom right). The left side of the STAR-CCM+ pipe is a mass flow inlet held at the same mass flow rate as the TRACE pipe. With each coupling iteration, information about the average non-inertial pressure gradient (denoted by $\langle\partial p / \partial x\rangle$ in Figure 9) is passed from CFD to TRACE in the form of a friction factor calculated by Eq. 10. The right side of the CFD model is held at a constant pressure, while the right and left sides of the TRACE model are connected to pipes as shown in Figure 9.

Domain decomposition coupling techniques are applied to the closed loop by removing the test section (outlined in Figure 9) from the TRACE model, using STAR-CCM+ for this region instead, and communicating pressure and mass flow data via two interfaces at either end of the CFD component as shown in Figure 9 (bottom left). The left side of the STAR-CCM+ component is a mass flow inlet 
receiving an interfacial mass flow rate $\left(\dot{m}_{i}\right)$ from TRACE and, in turn, providing a pressure $\left(p_{i}\right)$. The right side of the CFD component is a pressure boundary that receives a pressure $\left(p_{i}\right)$ from a FILL component (mass flow rate boundary) in TRACE and, in turn, provides a mass flow rate $\left(\dot{m}_{i}\right)$ to TRACE.

\subsubsection{Steady State}

First, TRACE standalone and coupled solutions using both domain decomposition and domain overlapping methodologies are compared for steady state cases. The resulting mass flow rates are plotted as functions of the pump rotation speed in Figure 10. The data all collapse onto approximately the same line, indicating that the CFD inlet profiles have minimal effect on the solution.

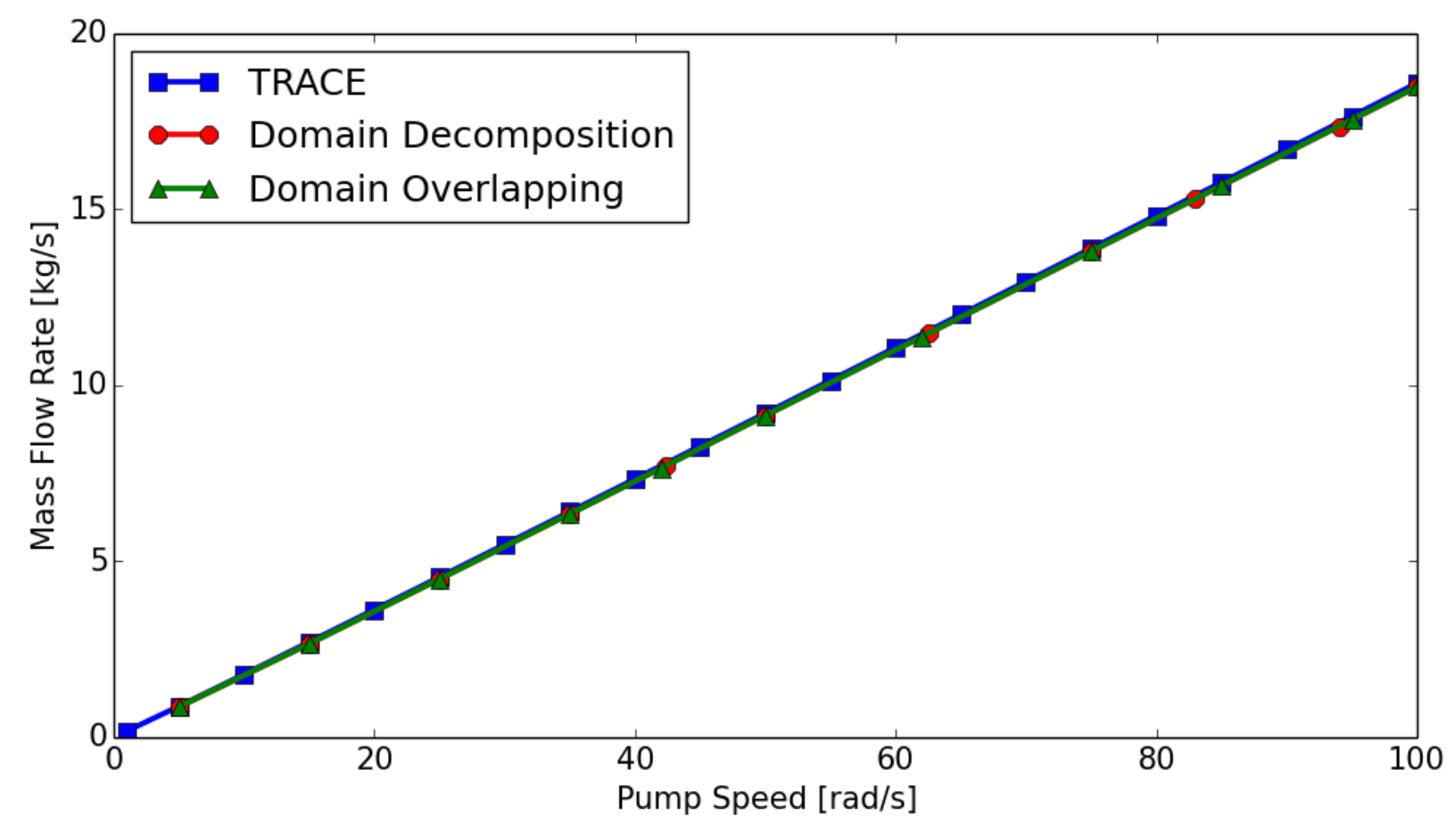

Figure 10. Steady state mass flow rates achieved via both coupling methodologies as compared to those calculated via TRACE standalone simulation.

We investigated the convergence performance of the two coupling methodologies by recording the number of iterations required to achieve convergence. The results are reported in Figure 11 for representative pump rotation rates. On the horizontal axis the guessed mass flow rate $\dot{m}_{\text {init }}$ at the beginning of the simulation is normalized by the final steady state flow rate $\dot{m}_{s s}$. The vertical axis displays the number of iterations required to achieve convergence. The $i^{t h}$ iteration is deemed converged if the criteria of Eq. 16 are met.

$$
\left|\dot{m}^{i}-\dot{m}^{i+1}\right|<\dot{m}^{i+1} \times 10^{-3} \text { and }\left|\dot{m}^{i+1}-\dot{m}^{i+2}\right|<\dot{m}^{i+1} \times 10^{-3}
$$




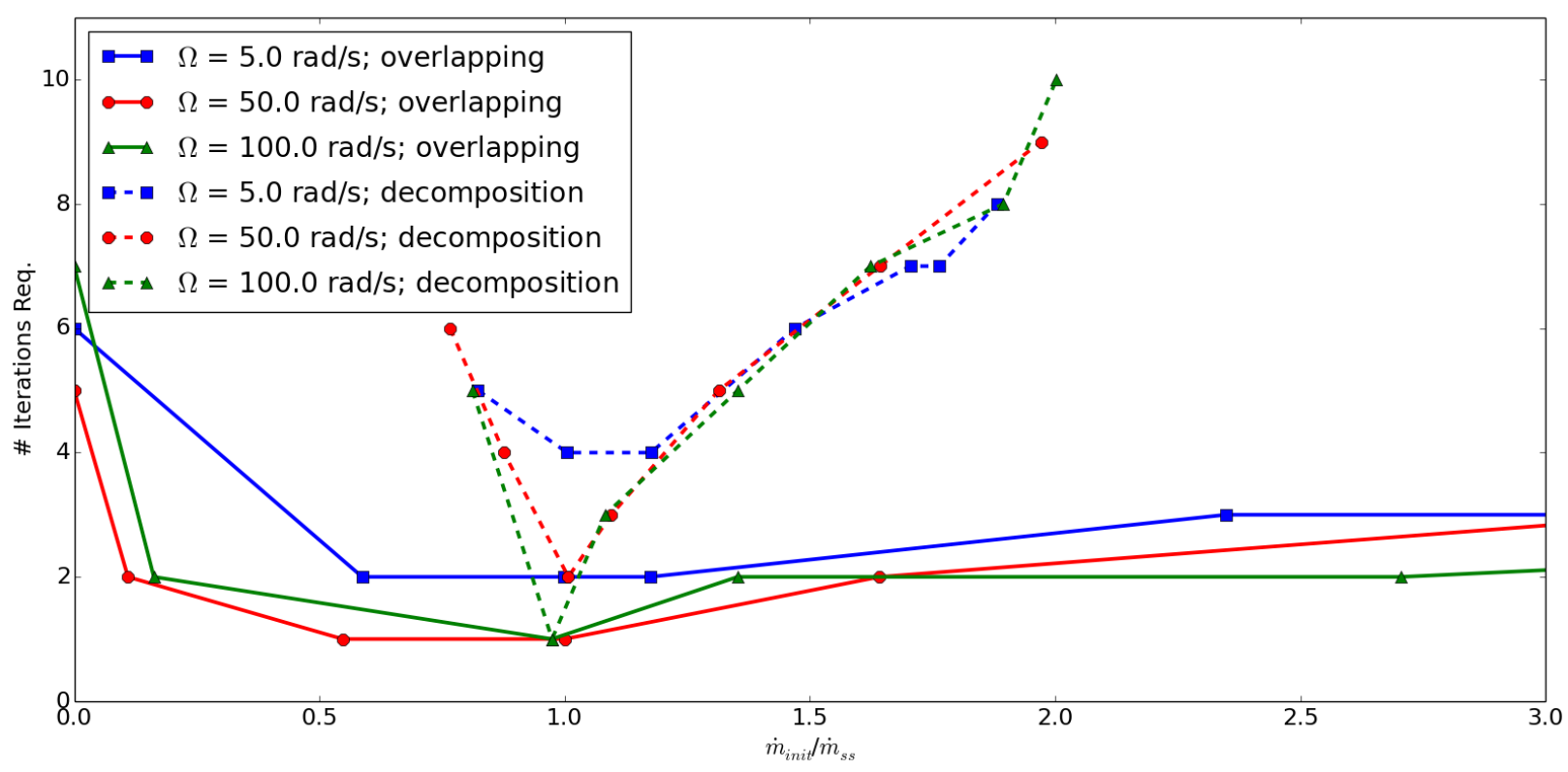

Figure 11. Iterations required for convergence of 6 coupling scenarios with varied initial mass flow rates. Solid lines refer to domain overlapping; dashed lines refer to domain decomposition. Three representative pump rates are indicated by the line color.

The initial mass flow rate was applied to domain decomposition cases by setting the values at the STARCCM+ inlet and the TRACE FILL component. In the domain overlapping cases, the values were only required in the STAR-CCM+ input. The domain decomposition coupling was not found to converge in all cases, and the data shown in Figure 11 approximately represent the range over which convergence was achieved. Identical TRACE numerical settings were used in both cases (i.e. convergence criteria and maximum pressure iterations both left as default). In contrast, the domain overlapping coupling was found to converge for all cases tested, demonstrating the superior robustness of this coupling methodology. Not only was the domain overlapping method less sensitive to initial guesses, but it also achieved convergence in fewer iterations for most conditions.

The less favorable performance of the domain decomposition approach results from the inability of TRACE to calculate a system wide pressure balance within a single coupled iteration, requiring several iterations for the system pressure to equilibrate. The domain decomposition simulations require multiple iterations for even the best initial mass flow rates, due to the effects of imperfect initial pressures set at the interface. Initial interface pressures were set to approximately correct values, but even small perturbations in the steady state pressure profiles requires additional iteration. This process is evidently exacerbated for low flow regimes in which the pressure drop is more sensitive to the flow rate. With starting conditions near $\dot{m}_{\text {init }} / \dot{m}_{s s} \approx 2.0$, the pressure rebalancing becomes too unstable and the TRACE iteration fails. To be sure, under-relaxation would improve convergence in these cases, but this is not considered here as the domain overlapping method required no such assistance. A further benefit of the domain overlapping method is that it depends only upon changes in pressure and not absolute pressure at the interface, eliminating a dimension of sensitivity.

\subsubsection{Transient}

With steady state convergence data in hand, the question of transient performance is now addressed. The pump impeller rotation rate was pulsed according to the function in Equation 17, designed to be smooth up to at least the first derivative. The mass flow rate through the loop and the pressure drop across the coupled test section (highlighted in Figure 9) were recorded as system response functions. 


$$
\Omega(t)=\left\{\begin{array}{c}
50.0 \frac{\mathrm{rad}}{\mathrm{s}}, \quad t \leq 100.0 \mathrm{~s} \\
50.0\left[1+\sin ^{4}\left(\frac{t-100.0 \mathrm{~s}}{10.0 \mathrm{~s}} \pi\right)\right] \frac{\mathrm{rad}}{\mathrm{s}}, 100.0 \mathrm{~s}<t<110.0 \mathrm{~s} \\
50.0 \frac{\mathrm{rad}}{\mathrm{s}}, \quad t \geq 110.0 \mathrm{~s}
\end{array}\right.
$$

Response functions for the mass flow rate and pressure drop across the test section are shown in Figure 12 alongside temporal convergence data. As expected, the mass flow rate appears to converge to a function resembling the pump impeller rotation rate, while the pressure drop converges to a function resembling the time derivative of the mass flow rate. An unphysical secondary pressure peak is observed for large integration time steps $(\Delta t \gtrsim 1.0 \mathrm{~s})$.
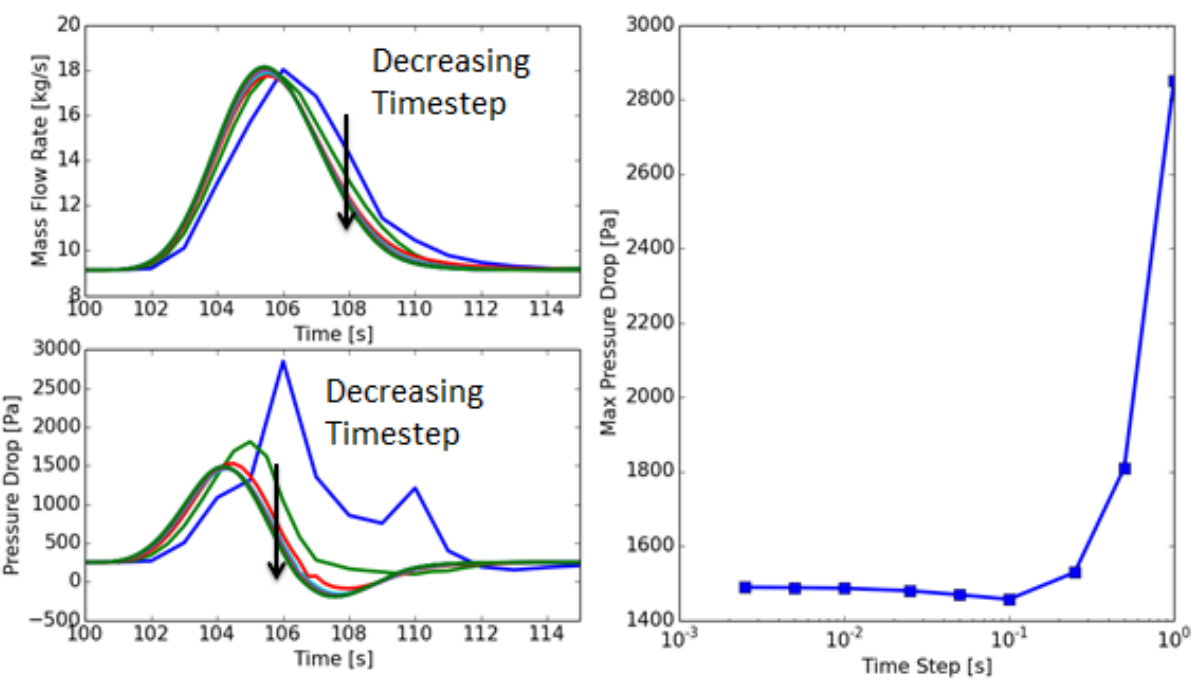

Figure 12. Transient response functions (left) and temporal convergence data (right) for domain decomposition coupling pump driven close loop test case. Multiple time steps are shown with the trend of decreasing time steps indicated.

The maximum pressure drop is plotted on the right of Figure 12 for each time step tested, giving an indication of the temporal convergence of the pressure response function. Significant error reduction is evident in the region $\Delta t \in\left[10^{-1} s, 10^{0} \mathrm{~s}\right]$, while the reduction drops off markedly for $\Delta t<10^{-1} \mathrm{~s}$.

The results for the domain overlapping coupled model and corresponding convergence data are shown in Figure 13. The unphysical secondary peak in the pressure drop of Figure 12 is not observed in this case for any time step used. 

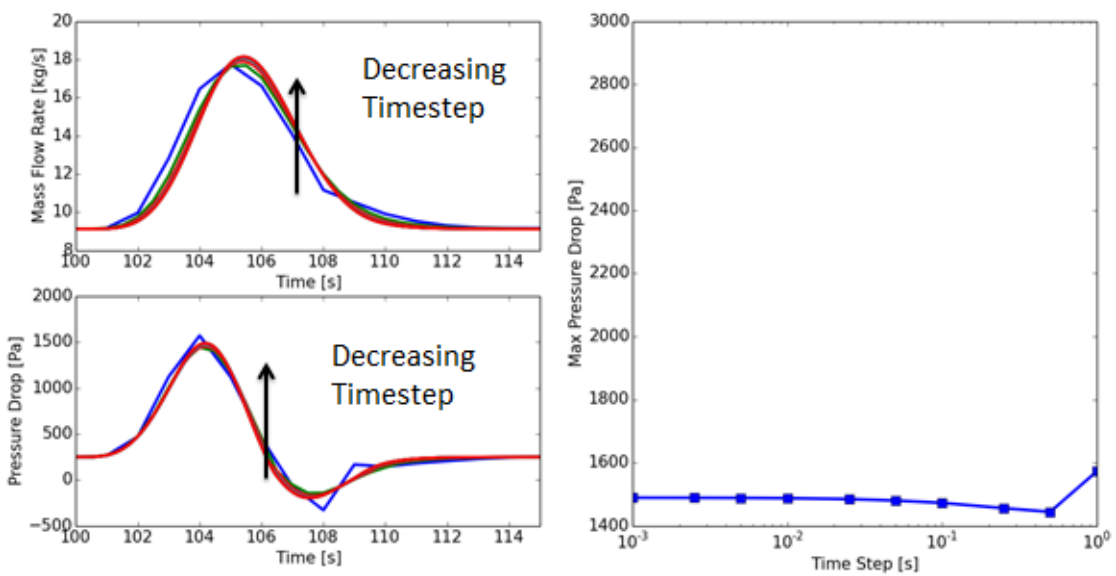

Figure 13. Mass flow rate and pressure drop response functions alongside temporal convergence data for the domain overlapping pump driven close loop transient test case. Multiple time steps are shown with the trend of decreasing time steps indicated.
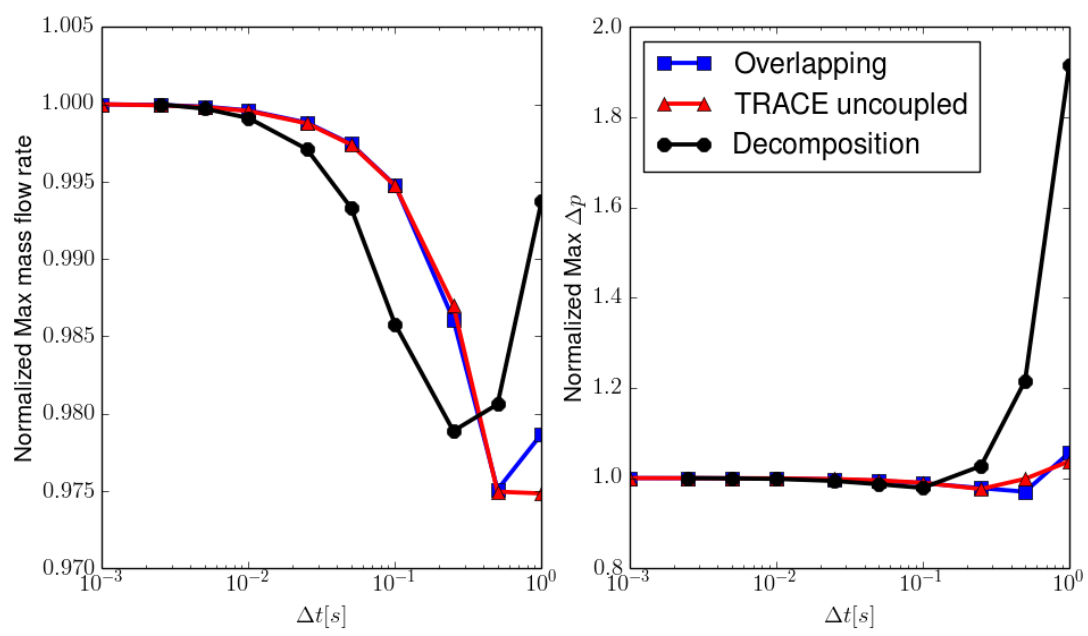

Figure 14. Plots of (normalized) maximum values of mass flow rate (left) and pressure drop (right) across the coupled section against the time step used for each transient case tested.

The comparison between TRACE standalone simulations and the two coupling methodologies is presented in Figure 14. The maximum values of mass flow rate and pressure drop are plotted against time step used for the cases tested in Figure 14. As expected, the converged solution of domain decomposition and domain overlapping is identical (Figure 14 left). This value is somewhat higher than the TRACE standalone case due to the developing flow conditions at the CFD inlet. In this case, the difference allows for visual verification that the domain overlapping method is implemented correctly, considered a positive side effect by the authors. The domain decomposition coupling requires smaller time steps to converge and produces highly inaccurate results for large time steps. Additionally, we found that the domain decomposition simulations became unstable for $\Delta t=1 \mathrm{~ms}$. At this level, perturbations to the velocity cause the inertial velocity derivative to become unstable as discussed below in §3.3.4. Remarkably, the convergence of the coupled solution when using domain overlapping methods is limited only by the convergence of TRACE, as can be observed in Figure 14. 


\subsubsection{Domain Decomposition Stability}

To consider the cause of the domain decomposition instability observed at small time steps in the previous section, consider once more the 1D model given by Eq. 11. In one iteration, STAR-CCM+ calculates a new pressure drop, estimated in Eq. 18, based on the previous velocity and the velocity just passed to it. For this analysis, we linearize the friction term based on the initial velocity $V_{0}$. The transient calculation of the previous section were preceded by several seconds of null transient with a mass flow rate of approximately $9.13 \mathrm{~kg} / \mathrm{s}$, making $V_{0}$ simple to calculate.

$$
\Delta p_{C F D}=2 f_{C F D} \frac{L_{C F D}}{D} \rho V^{n} V_{0}+\frac{\rho L_{C F D}}{\Delta t}\left(V^{n+1}-V^{n}\right)
$$

where $f_{c c m}$ is a friction factor characterizing the frictional losses of the CFD portion of the simulation. Using this pressure drop, the next time step velocity calculated by TRACE is estimated with Eq. 19

$$
\rho L_{T R C} \frac{V^{n+2}-V^{n+1}}{\Delta t}=\Delta p_{C F D}-2 f_{T R C} \frac{L_{T R C}}{D} \rho V^{n+1} V_{0}
$$

Combining Eqs. 18 and 19 yield the recurrence relation shown in Eq. 20.

$$
V^{n+2}=V^{n+1}\left(1+\frac{L_{C F D}}{L_{T R C}}-\frac{2 f_{T R C} \Delta t}{D} V_{0}\right)+V^{n} \frac{L_{C F D}}{L_{T R C}}\left(\frac{2 f_{C F D} \Delta t}{D} V_{0}-1\right)
$$

Performing a rudimentary Von Neumann stability analysis $\left(V^{n+1}=V^{n} e^{\lambda \Delta t}\right)$ yields the error amplification factor of Eq. 21.

$$
G \equiv \frac{V^{n+1}}{V^{n}}=e^{\lambda \Delta \mathrm{t}}=\frac{L_{C F D}}{L_{T R C}}\left(1+\frac{L_{C F D}}{L_{T R C}}-\frac{2 f_{T R C} \Delta t}{D} V_{0}\right)\left(\frac{2 f_{T R C} \Delta t}{D} V_{0}-1\right)
$$

While this model is too crude to accurately predict the stability bounds, it does predict that stability $(|G| \leq 1)$ requires that the time step used fall within a specific range, since $G$ is quadratic in $\Delta t$. The upper bound of this range represents the familiar stability limit of explicit time stepping, while the lower bound represents a limit at which perturbations to the velocity cause unstably large perturbations to the inertial term. The domain overlapping method was found to be stable for all timesteps tested.

\section{CONCLUSIONS}

In this paper, a novel domain overlapping method is presented for the coupling of CFD and 1D system codes. The method allows on-the-fly correction of non-inertial pressure drops in the momentum equations of the 1D code, based on the CFD solution which is markedly more accurate under certain flow conditions. The performance of the novel method is compared to a domain decomposition coupling scheme using a variety of test cases. Consistency, stability, and convergence characteristics of the two coupling methods are compared with the objective of identifying strengths and weaknesses.

The proposed domain overlapping method proved feasible and accurate for all cases tested, both in steady state and transient conditions. Results showed that the developed domain overlapping coupling method exhibits superior convergence and numerical stability when compared to the domain decomposition approach. We showed that domain decomposition coupling results in a geometric discrepancy that requires additional iterations to overcome. In transient mode, this discrepancy can disrupt the entire simulation and result in unphysical results for large time steps. Additionally, since the domain overlapping method relies on changes in pressure rather than absolute pressures, a dimension of sensitivity is avoided. Conversely, the implementation of a domain decomposition approach is significantly easier than domain overlapping methods and automatically enforces consistency between the two codes. 
Most modern best-estimate system codes also include a pseudo-3D formulation of the momentum equation to model 3D components such as the reactor pressure vessel. These components are instrumental in the simulation of reactor transients. While additional complexity is added by the inability to easily define cross section averaged flow paths, many of the ideas presented here are extensible to 3D geometries. The extension of our domain overlapping approach to the coupling with system code 3D components will be reported in an upcoming paper.

The usefulness of any CFD-STH coupling relies on the assumption that CFD produces superior results for the coupled region. However, STH codes employ correlations derived from experimental data. In order to truly justify a coupled simulation, validation exercises must be carried out showing that the CFD method exhibits superior accuracy. Future work on this project will therefore require detailed comparisons of CFD results and experimental data.

\section{ACKNOWLEDGMENTS}

This work was supported through a DOE NEUP fellowship and the US NRC grant No. NRC-HQ-12-G04-0083.

\section{REFERENCES}

[1] U. S. Nuclear Regulatory Commission, TRACE V5.0 Theory Manual, 2010.

[2] INL, "RELAP5-3D Code Manuals Revision 2.3," Idaho National Lab, Idaho Falls, ID, 2005.

[3] D. Tenchine, R. Baviere, P. Bazin, F. Ducros, G. Geffraye, D. Kadri, F. Perdu, D. Pialla, B. Rameau and N. Tauveron, "Status of CATHARE code for sodium cooled fast reactors," Nuclear Engineering and Design, vol. 245, pp. 140-152, 2012.

[4] G. Lerchel, H. Austregesilo, P. Schöffel, D. von der Cron and F. Weyermann, "ATHLET mod 3.0, Cycle A, User's Manual GRS-p1/Vol1, Rev.6," GRS, Germany, 2012.

[5] T. Höhne, S. Kliem, U. Rohde and F.-P. Weiss, "Boron Dilution Transients During Natural Circulation Flow in PWR-Experiments anad CFD Simulations," Nuclear Engineering and Design, vol. 238, pp. 1987-1995, 2008.

[6] G. H. Lee, Y. S. Bang, S. W. Woo and A. J. Cheong, "Comparative study on the effect of reactor internal structure geometry modeling methods on the prediction accuracy for PWR internal flow distribution," Annals of Nuclear Energy, vol. 70, pp. 208-215, 2014.

[7] J. H. Jeong and B.-S. Han, "Coolant Flow Field in a Real Geometry of PWR Downcomer and Lower Plenum," Annals of Nuclear Energy, vol. 35, pp. 610-619, 2008.

[8] E. Merzari and H. Ninokata, "Proper orthogonal decomposition of the flow in a tight lattice rodbundle," Nuclear Engineering and Design, vol. 241, pp. 4621-4632, 2011.

[9] D. P. Prill and A. G. Class, "Semi-automated proper orthogonal decomposition reduced order model non-linear analysis for future BWR stability," Annals of Nuclear Energy, vol. 67, pp. 70-90, 2014.

[10] D. L. Aumiller, E. T. Tomlinson and R. C. Bauer, "A Coupled RELAP5-3D/CFD Methodology with a Proof-of-Principle Calculation," Nuclear Engineering and Design, vol. 205, pp. 83-90, 2001.

[11] H. Gibeling and J. Mahaffy, "Benchmarking Simulations with CFD to 1-D Coupling," Penn State University, Applied Research Laboratory, 2002.

[12] N. Anderson, Y. Hassan and R. Schultz, "Analysis of the hot gas flow in the outlet plenum of the very high temperature reactor using coupled RELAP5-3D system code and a CFD code," Nuclear Engineering and Design, vol. 238, pp. 274-279, 2008.

[13] D. Bertolotto, A. Manera, S. Frey, H.-M. Prasser and R. Chawla, "Single-phase mixing studies by 
means of a directly coupled CFD/system-code tool," Annals of Nuclear Energy, vol. 36, pp. 310-316, 2009.

[14] A. Papukchiev, G. Lerchl, C. Waata and T. Frank, "Extension of the Simulation Capabilities of the 1D System Code ATHLET by Coupling with the 3D CFD Software Package ANSYS CFX," in Proc. The 13th International Topical Meeting on Nuclear Reactor Thermal Hydraulics (NURETH-13), Kanazawa City, Ishikawa Prefecture, Japan.

[15] A. Papukchiev, G. Lerchl, J. Weis, M. Scheuerer and H. Austregesilo, "Development of coupled 1D3D thermal-hydraulic code for nuclear power plant simulation and its application to a pressurized thermal shock scenario in PWR," in Proceedings of The 14th International Topical Meeting on Nuclear Reactor Thermalhydraulics, NURETH-14, Toronto, Ontario, Canada, 2011.

[16] T. Watanabe, Y. Anoda and M. Takano, "System-CFD coupled simulations of flow instability in steam generator U tubes," Annals of Nuclear Energy, vol. 70, pp. 141-146, 2014.

[17] W. Li, X. Wu, D. Zhang, G. Su, W. Tian and S. Qiu, "Preliminary study of coupling CFD code FLUENT and system code RELAP5," Annals of Nuclear Energy, vol. 73, pp. 96-107, 2014.

[18] T. H. Fanning and J. W. Thomas, "Advances in Coupled Safety Modeling Using Systems Analysis and High-Fidelity Methods," ANL-GENIV-134, 2010.

[19] M. Jeltsov, K. Kööp, P. Kudinov and W. Villanueva, "Development of a Domain Overlapping Coupling Methodology for STH/CFD Analysis of Heavy Liquid Metal Thermal-Hydraulics," in Proc. The 15th International Topical Meeting on Nuclear Reactor Thermal Hydraulics (NURETH15), Pisa, Italy, 2013.

[20] R. Bavière, N. Tauveron, F. Perdu, E. Garrè and S. Li, "A first system/CFD coupled simulation of a complete nuclear reactor transient using CATHARE2 and TRIO_U. Preliminary validation on the Phénix Reactor Natural Circulation Test," Nuclear Engineering and Design, vol. 277, pp. 124-137, 2014.

[21] D. Pialla, D. Tenchine, S. Li, P. Gauthe, A. Vasile, R. Baviere, N. Tauveron, F. Perdu, L. Maas, F. Cocheme, K. Huber and X. Cheng, "Overview of the system alone and system/CFD coupled calculations of the PHENIX Natural Circulation Test within the THINS project," Nuclear Engineering and Design, vol. 290, pp. 78-86, 2015.

[22] F. Cadinu and P. Kudinov, "Development of a "Coupling-by-Closure" Approach between CFD and System Thermal-Hydraulic Codes," in Proc. The 13th International Topical Meeting on Nuclear Reactor Thermal Hydraulics (NURETH-13), Kanazawa City, Ishikawa Prefecture, Japan, 2009.

[23] A. Papukchiev, M. Jeltsov, K. Kööp, P. Kudinov and G. Lerchl, "Comparison of different coupling CFD-STH approaches for pre-test analysis of TALL-3D experiment," Nuclear Engineering and Design, vol. 290, pp. 135-143, 2015.

[24] G. Bandini, M. Polidori, A. Gerschenfeld, D. Pialla, S. Li, W. M. Ma, P. Kudinov, M. Jeltsov, K. Kööp, K. Huber, X. Cheng, C. Bruzzese, A. G. Class, D. P. Prill, A. Papukchiev, C. Geffray, R. Macian-Juan and L. Maas, "Assessment of Systems Codes and their Coupling with CFD Codes in Thermal-Hydraulic Applications to Innovative Reactors," Nuclear Engineering and Design, vol. 281, pp. 22-38, 2015.

[25] T. L. Bergman, A. S. Lavine, F. P. Incropera and D. P. Dewitt, Fundamentals of Heat and Mass Transfer: Seventh Edition, John Wiley \& Sons, Inc., 2011.

[26] J. H. Mahaffy, "A Stability-Enhancing Two-Step Method for Fluid Flow Calculations," Journal of Computational Physics, vol. 46, pp. 329-341, 1982.

[27] D. Bertolotto, Coupling a System Code with Computational Fluid Dynamics for the Simulation of Complex Coolant Reactivity Effects, École Polytechnique Fédérale de Lausanne, 2011. 